\title{
Very late complications of oncotherapy in glioblastoma patients: A case series
}

\author{
Ondrej Kalita ${ }^{a}$, Lumir Hrabaleka, Matej Halaja, Pavel Hok ${ }^{b}$, David Franc ${ }^{b}$, Yvona Klementovac, Martin Dolezel', \\ Eva Cechakova ${ }^{d}$, Zuzana Sporikova ${ }^{e}$, Jiri Drabek ${ }^{e}$, Marian Hajduch ${ }^{e}$, Lucie Tuckova ${ }^{f}$
}

Background. Stroke-like syndrome is defined as a rare, delayed complication of brain oncotherapy. Cases with more favorable brain cancer diagnoses and longer life expectancy have been previously reported, but here we present, for the first time, three long-term survivors of glioblastoma with stroke-like syndromes.

Methods and Results. Three young or middle-aged patients underwent tumor resection and chemoradiotherapy. They received regular clinical and imaging follow-up with stable neurological status and no signs of tumor recurrence. They exhibited varied signs and symptoms (motor and sensory deficits, aphasia, memory and cognitive disorders, seizures, and headache) accompanied by imaging abnormalities. Stroke-like syndromes developed within 2-5 days and resolved in 2-6 weeks. Diffusion-weighted MRI and T2 brain perfusion abnormalities were demonstrated in all patients. In addition, there was focal T1 MRI contrast enhancement due to blood-brain barrier disruption. In addition to tumor recurrence, classic stroke, encephalitis, metabolic and mitochondrial disorders, and post-seizure swelling should be excluded. The imaging indicated intensive MRI scanning and symptomatic medication (steroids supplemented by antiepileptics, vasoactive agents, etc.) for judicious management. With respect to the course, an invasive procedure was still considered an option.

Conclusion. All stroke-like syndromes are diagnoses of exclusion. To avoid misinterpretation of imaging findings as glioblastoma recurrence and avert recall oncotherapy or redundant interventions, better understanding of delayed complications of brain tumor therapy is crucial.

Key words: stroke-like syndrome, glioblastoma, oncotherapy, corticosteroid

Received: November 28, 2020; Revised: January 14, 2021; Accepted: January 29, 2021; Available online: February 22, 2021 https://doi.org/10.5507/bp.2021.012

(c) 2022 The Authors; https://creativecommons.org/licenses/by/4.0/

${ }^{a}$ Department of Neurosurgery, University Hospital Olomouc and Faculty of Medicine and Dentistry, Palacky University Olomouc, Czech Republic

${ }^{b}$ Department of Neurology, University Hospital Olomouc and Faculty of Medicine and Dentistry, Palacky University Olomouc, Czech Republic 'Department of Oncology, University Hospital Olomouc and Faculty of Medicine and Dentistry, Palacky University Olomouc, Czech Republic ${ }^{d}$ Department of Radiology, University Hospital Olomouc and Faculty of Medicine and Dentistry, Palacky University Olomouc, Czech Republic eLaboratory of Experimental Medicine, Institute of Molecular and Translational Medicine, Faculty of Medicine and Dentistry, Palacky University Olomouc, Czech Republic

${ }^{f}$ Department of Pathology and Laboratory of Molecular Pathology, Institute of Molecular and Translational Medicine, Faculty of Medicine and Dentistry, Palacky University Olomouc, Czech Republic

Corresponding author: Ondrej Kalita: e-mail: ondrej.kalita@fnol.cz

\section{BACKGROUND}

Despite advanced imaging, surgical, and oncological techniques, the prognosis of patients with glioblastoma (GBM) remains dismal. However, some signs of improvement in treatment outcomes of patients with GBM can be traced over the last decades ${ }^{1}$.

The best outcomes are yielded by aggressive multimodal therapy involving safe and maximal resection followed by chemoradiotherapy ${ }^{1}$. However, side effects of this aggressive treatment seriously impact not only GBM patients' quality of life, but also their overall survival. The ensuing follow-up involves MRI and clinical status review, assessment of tumor recurrence or residuum, and distinguishing between true progression, pseudoresponse, and pseudoprogression. Temporary and permanent surgical complications are obvious immediately after resection and within several months, respectively. By contrast, side effects of oncological treatment commonly have a gradual presentation, some occurring after months or even years. In particular, very late complications are the most difficult to identify. Several diagnostic items have been defined.

"SMART" - stroke-like migraine attacks after radiotherapy is an uncommon, very late complication of cranial radiotherapy. It typically presents as reversible, unilateral cortical signs on MRI and symptoms such as confusion, hemiparesis, seizures, and headaches².

"PIPG" - peri-ictal pseudoprogression refers to transient seizure-related MRI changes that could mimic disease progression ${ }^{3}$.

"ALERT" - a syndrome characterized by acute lateonset encephalopathy after radiotherapy ${ }^{4}$. 


\section{CASE 1 - SMART}

In 2010, a 37-year-old woman presented with a two-week history of fluctuating left-sided hemiparesis and sensory symptoms. Brain MRI revealed a contrast-enhanced tumor in the parasagittal region of the right parietal lobe. She received radical resection in January 2010, followed by chemoradiotherapy (Stupp regime). Histological diagnosis confirmed GBM, immunohistochemistry detected IDH1 canonical R132H mutation, and PCR revealed MGMT promoter methylation and IDH1 canonical $\mathrm{R} 132 \mathrm{H}$ mutation, retrospectively ${ }^{5}$. She was followed by regular clinical and imaging check-ups, with stable neurological status and no signs of tumor recurrence. Despite her left hemiparesis, she had independent gait, and permanently looked after her blind husband. In January 2019, a follow-up MRI disclosed a new, contrast-enhanced lesion in the periventricular region of her right temporal lobe, relatively far from the original resection cavity (Fig. 1a,b). In February 2019, a PET FLT scan showed no radiotracer uptake in brain tissue (Fig. 1c). Surprisingly, another follow-up MRI showed effacement of the contrast-enhanced lesion in March 2019 (Fig. 1d). In May 2019, there was disability progression, with worsening of hemiparesis, accompanied by development of anxiety and depressed mood, migraine and mild epileptic seizures. A new MRI, in June 2019, revealed new multiloculated contrast-enhanced lesions around the original resection cavity in the right parietal lobe and a blurred contrast-enhanced region in the right temporal lobe (Fig. 1d). EEG showed a nonspecific abnormal pattern consistent with a lesion, with no signs of epileptic activity. The patient received antiepileptics, corticoids, and anxiolytics, rehabilitation services and psychiatric interventions. Another follow-up MRI, in September 2019, again showed lesion disappearance. Based on an incidental MRI finding, complementary CT angiography confirmed a radiation-related basilar tip aneurysm, which was managed with endovascular coil embolization (October 2019) (Fig. 1e,f). The next followup MRI (September 2020) detected no enhanced lesion (Fig. 1g,h). There was an incomplete recovery. The patient was free from migraine, anxiety, and epileptic seizures, but continued to use a wheelchair due to severe left hemiparesis.

\section{CASE 2 - SMART/PIPG}

In 2009, a 16-year-old male patient presented with a short history of right-sided hemiparesis. Brain MRI revealed a contrast-enhanced tumor in the left TP cerebral region. He underwent radical resection, followed by chemoradiotherapy (Stupp regime). The histological diagnosis was GBM, and retrospective immunohistochemical analysis detected IDH1 mutation. He was followed by regular clinical and imaging check-ups with no signs of tumor recurrence or neurological deterioration. He was fully independent, with no epileptic seizures, and no motor or sensory deficits. He graduated from the Faculty of Law of a local university in June 2018. In December 2018 he suffered from sudden onset of right-sided hemiparesis, hemihypesthesia, and speech disorder. Emergency MRI showed a hyposignal and hypersignal lesion in the frontal periphery of the resection cavity, visible in T1 and FLAIR scans, respectively (Fig. 2a,b), suggestive of acute ischemia. Accordingly, MR angiography revealed acute ischemia in the MCA region. PET FLT, echocardiography, Holter ECG, and extracranial vessel ultrasonography yielded no tumor recurrency or cardiovascular emboligenic perturbation. After conservative treatment (anticoagulant and antiplatelet therapy, anxiolytics) for a month, he recovered uneventfully. MRI revealed no tumor recurrence in May 2020.

\section{CASE 3 - PIPG/ALERT}

In 2012, a 39-year-old man presented with his first epileptic seizures. Brain MRI revealed a contrast-enhanced tumor in the parasagittal region of the right parietal lobe. He underwent radical resection, followed by chemoradiotherapy (Stupp regime). The histological diagnosis was GBM. Retrospective immunohistochemical analysis detected canonical R132H mutation of IDH1, and PCR revealed MGMT promoter methylation and canonical R132H mutation of IDH1 (ref. ${ }^{5}$ ). He was followed by regular clinical and imaging check-ups with no signs of tumor recurrence or neurological deterioration. Despite only partial epileptic control (with a few remaining mild episodes per month), he was independent, with no mobility impairment. In January 2018, disclosure by a follow-up MRI of a new, blurred, small contrast-enhanced lesion in the resection cavity wall and the adjacent cortex was followed by a rise in convulsive activity (Fig. 3a,b). A PET FLT scan showed no radiotracer uptake in brain tissue. His clinical status stabilized following modification of antiepileptic therapy and corticoid administration. The patient recovered completely. MRI indicated no tumor recurrence in June 2020 (Fig. 3c).

\section{DISCUSSION}

All our patients shared common features, including a radically resected, IDH-mutated, and MGMT-methylated GBM, a history of chemoradiotherapy, young to middle age tumor presentation, and long survival. For a long period in the regular follow-up, they were in relatively good health and showed no evidence of tumor recurrence. Both patients 1 and 3 had a stable, radiation-induced leukoencephalopathy. The onset of neurological deterioration was characterized by ambiguous dynamics. The new, transient, diffuse, cortical postcontrast abnormalities on MRI in Patients 1 and 3 preceded episodes of headaches, drowsiness, confusion/hallucinations, stroke-like symptoms, and complex seizures for several months. By contrast, Patient 2 suffered from post-stroke neurological impairment without prodromal MRI abnormalities. Due to the clinical severity of their status, Patients 1 and 2 required hospital admission. Initial MRI changes mimicked some aspects of GBM progression, with edema and gadolinium- 


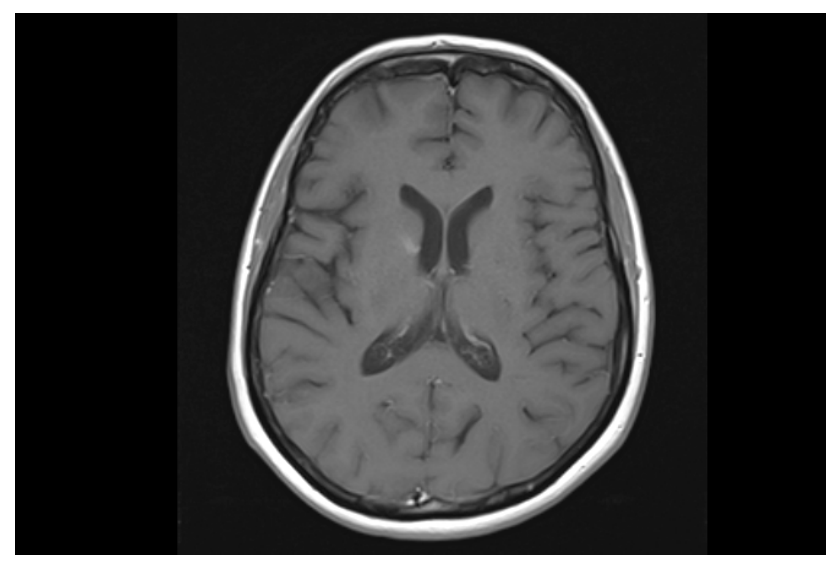

Fig. 1a. Axial T1-weighted MRI scan, showing contrast-enhanced lesion in the periventricular region of the right temporal lobe.

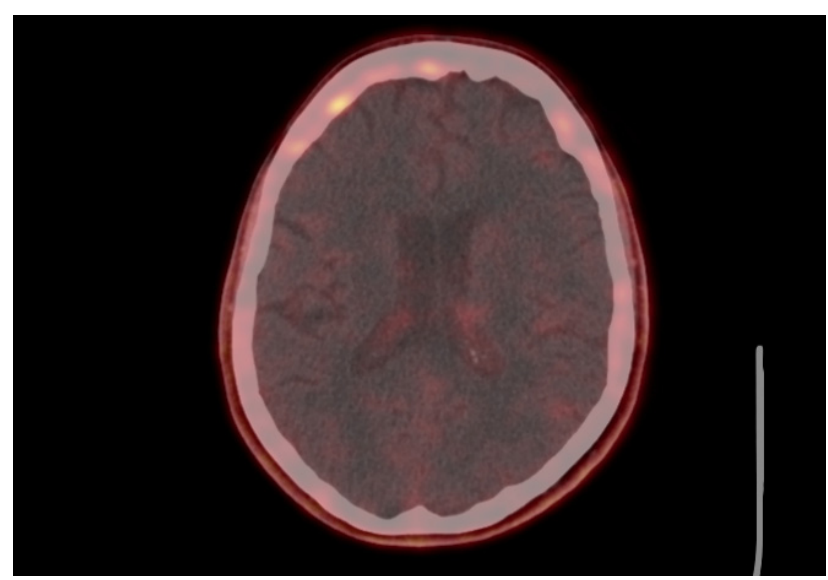

Fig. 1c. PET FLT scan showing no radiotracer uptake in brain tissue.

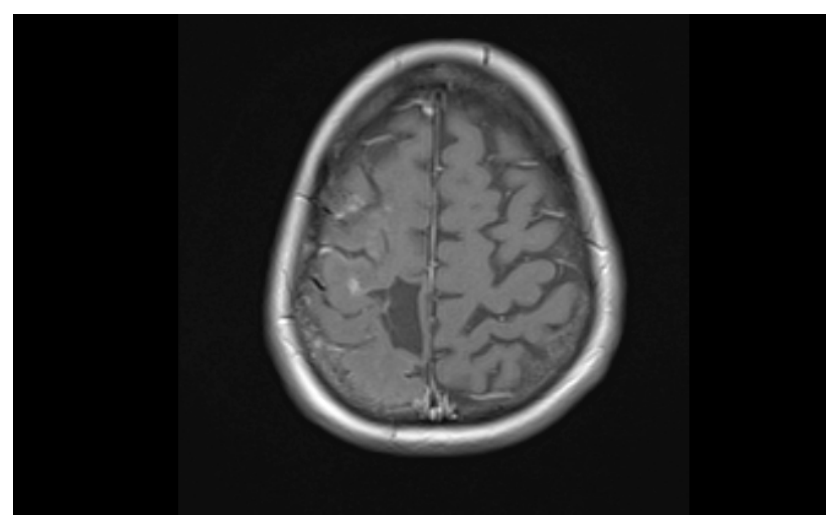

Fig. 1e. Axial T1-weighted MRI scan showing multiloculated contrast-enhanced lesions around the original resection cavity in the right parietal lobe and a blurred contrast-enhanced region in the right temporal lobe.

enhancing foci, including the cortex. However, although standard T1 postcontrast, diffusion imaging and PET FLT scans showed uncommon or elusive GBM features, the MRI abnormalities were limited in brain tissues near or not far from the resection cavity. Results of CSF analysis (cell count, protein transfer, total proteins, glucose,

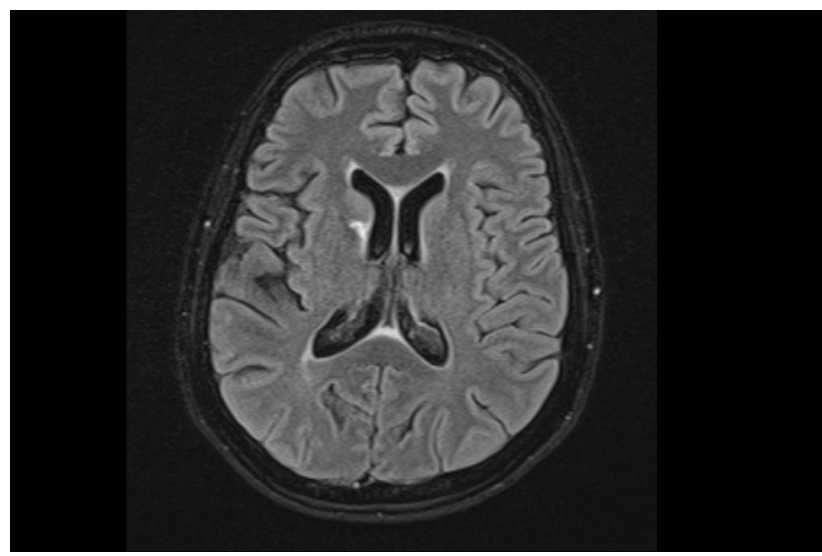

Fig. 1b. Diffusion-weighted MRI showing local hyperintensity.

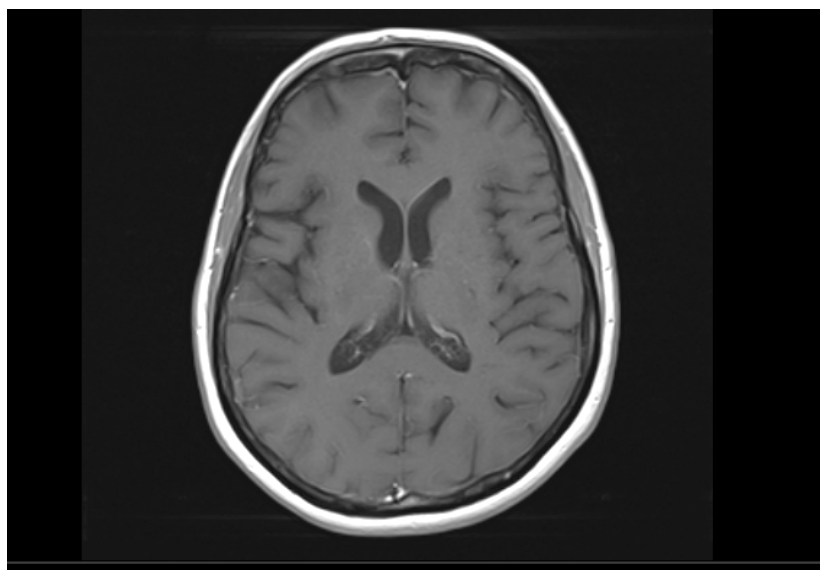

Fig. 1d. Axial T1-weighted MRI scan showing effacement of the contrast-enhanced lesion in the right periventricular region.

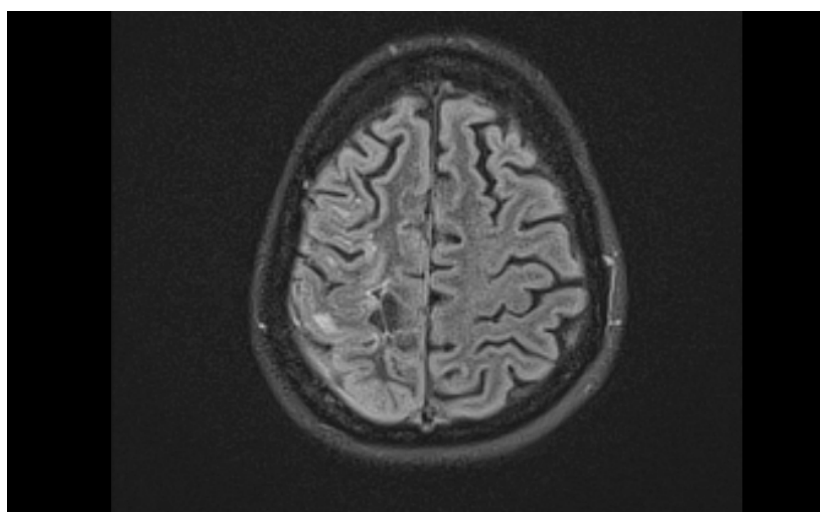

Fig. 1f. Diffusion-weighted MRI scan showing multilocal hyperintensity.

PCR-based examination of CSF for viral infection) were unremarkable for all patients. EEG recordings during the acute phase showed diffuse slow abnormalities in all patients. To avoid burdensome diagnostic and therapeutic procedures, an intensive wait-and-scan strategy was adopted. There is no causal treatment, so symptomatic 


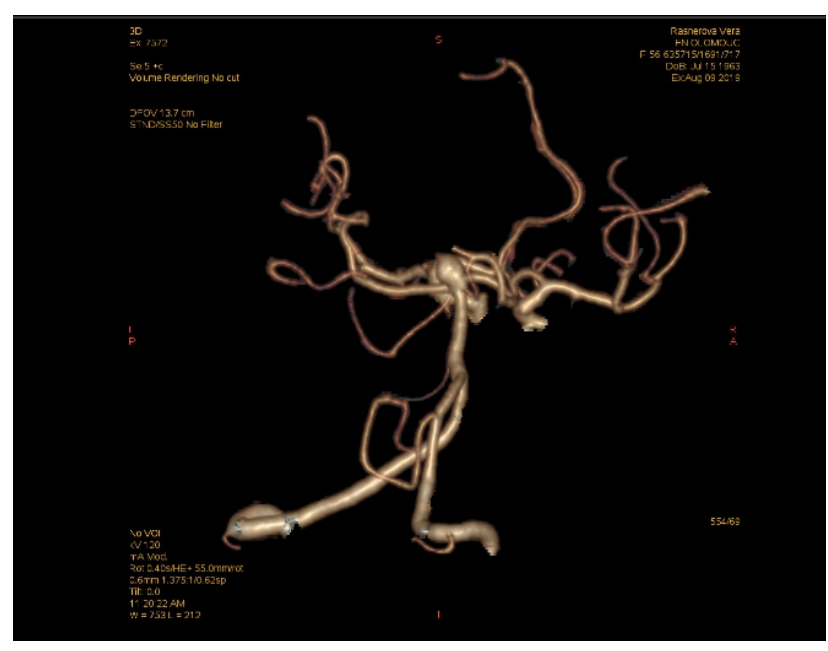

Fig. 1g. CT angiograph confirming a radiation-related basilar tip aneurysm that was managed with endovascular coil embolization (1h).

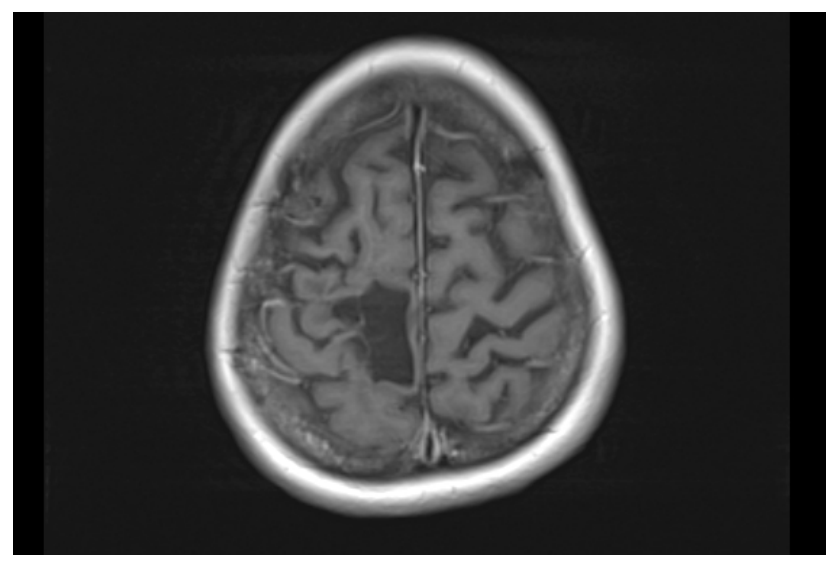

Fig. 1i. Axial T1-weighted MRI scan showing disappearance of the multiloculated contrast-enhanced lesions.

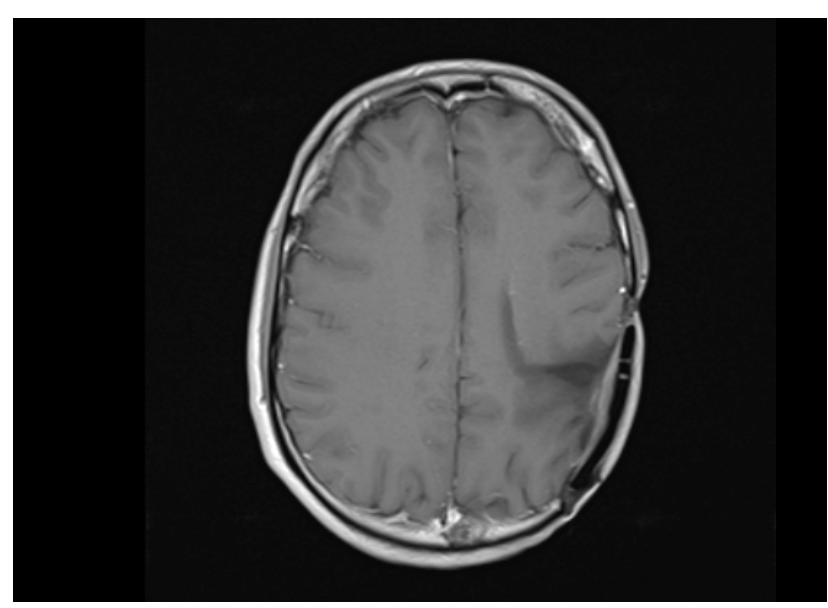

Fig. 2a. Axial T1-weighted MRI scan showing no contrastenhanced lesion.

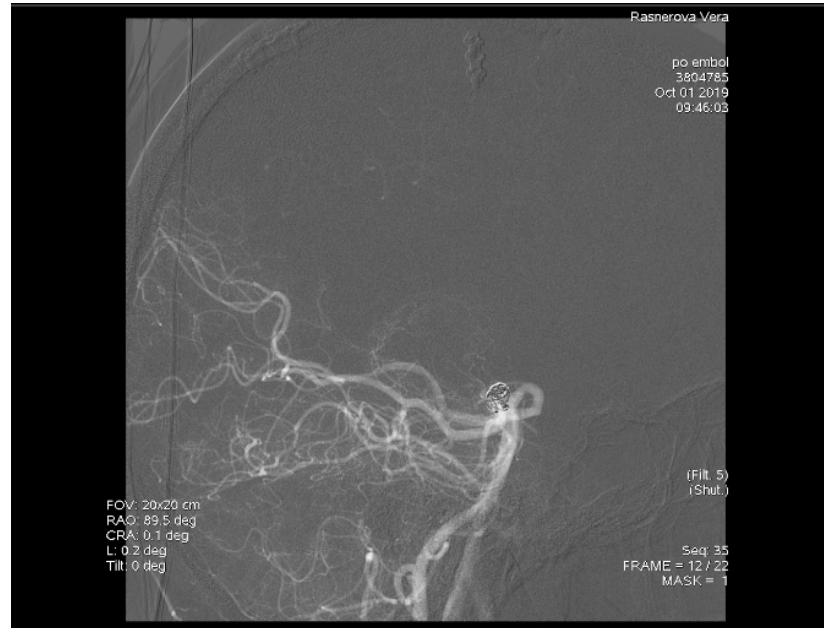

Fig. 1h. Endovascular coil embolization.

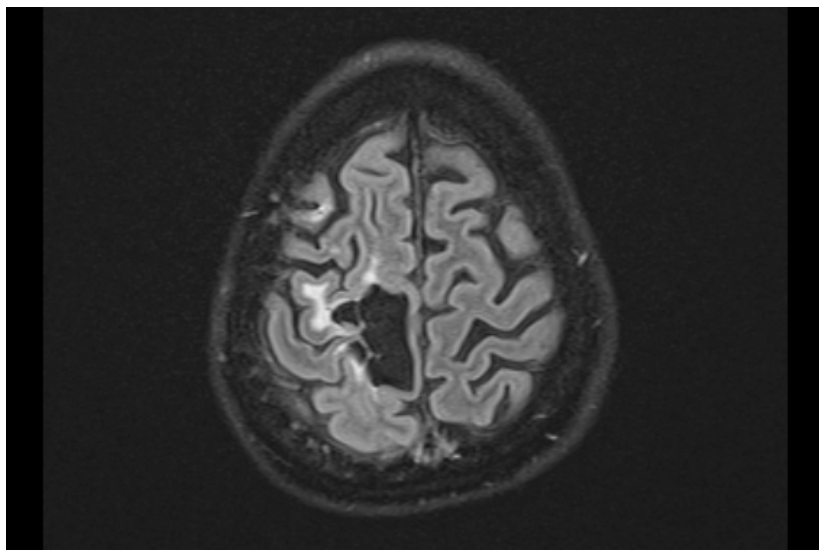

Fig. 1j. Axial diffusion-weighted MRI scan showing reduction in multilocal hyperintensity.

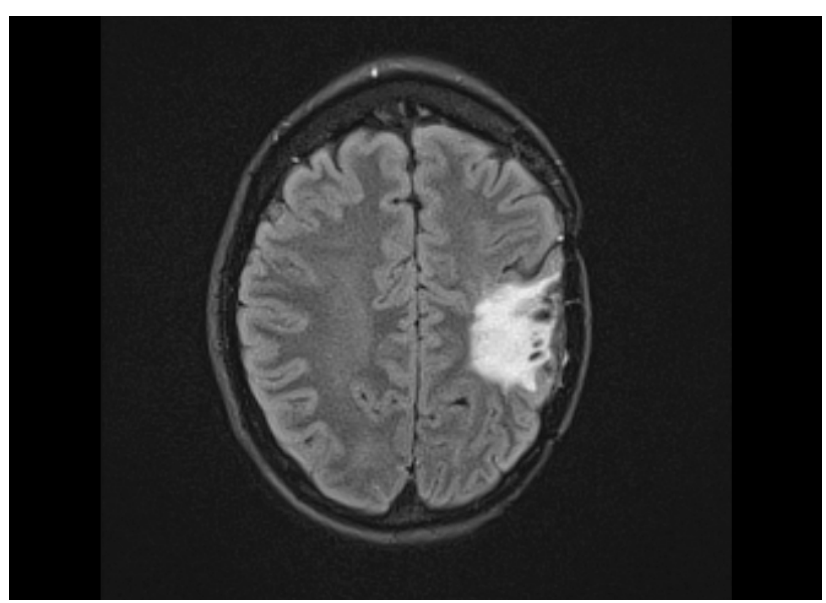

Fig. 2b. Diffusion-weighted MRI scan showing hypersignal zone in the frontal periphery of the resection cavity. 


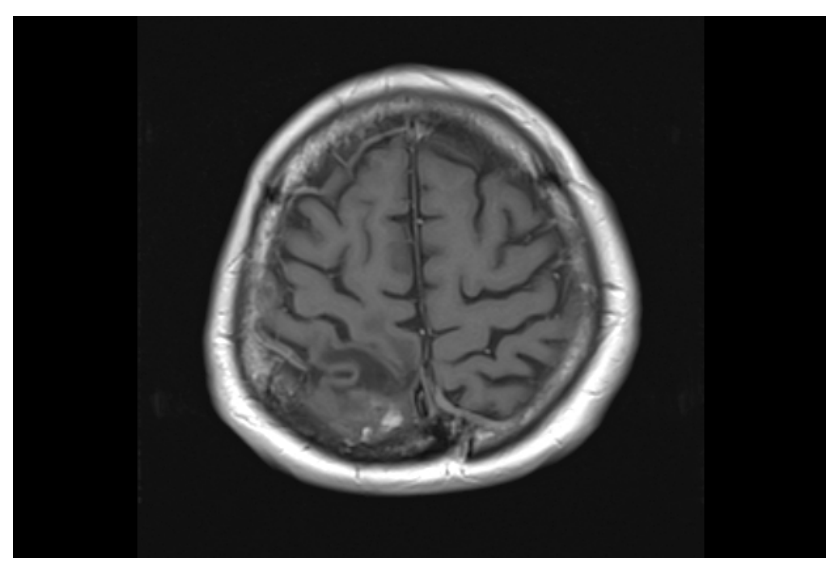

Fig. 3a. Axial T1-weighted MRI scan showing contrast-enhanced lesion around the original resection cavity in the right parietal lobe.

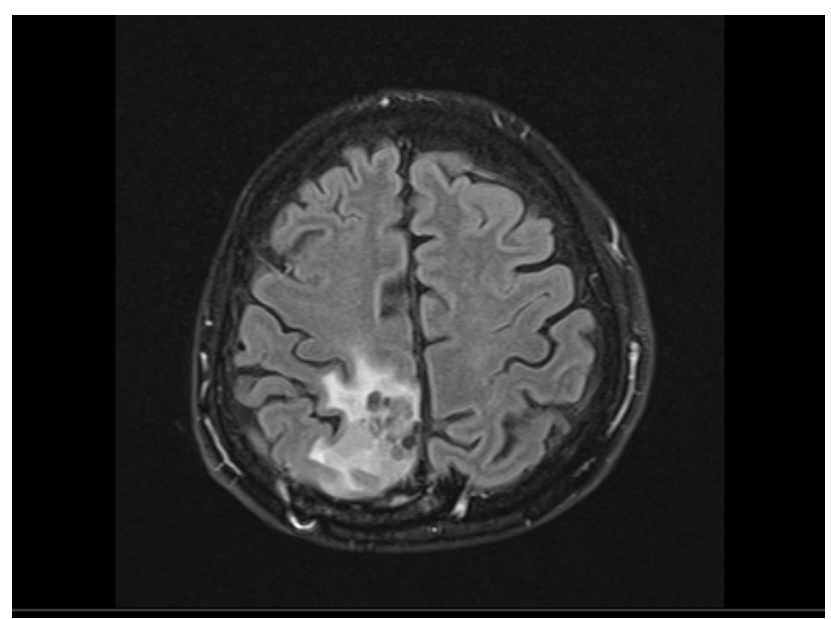

Fig. 3b. Diffusion-weighted MRI scan showing multilocal hypersignal rim encompassing the resection cavity shown in $3 \mathrm{a}$.

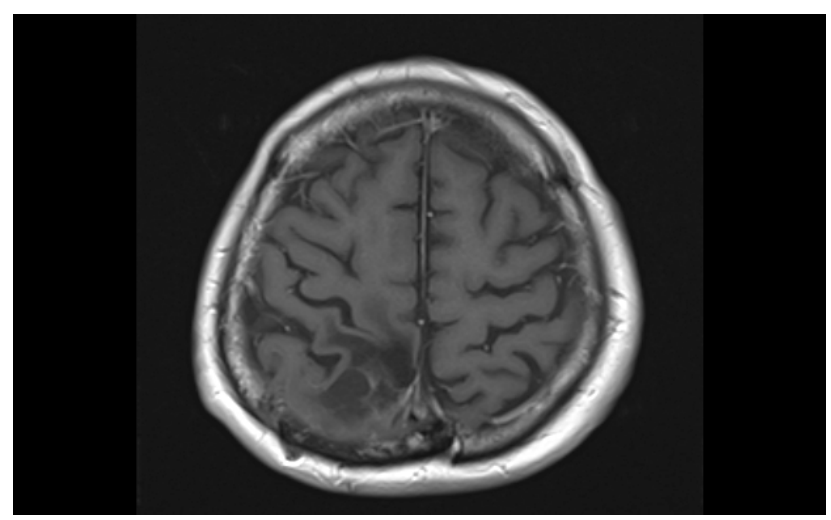

Fig. 3c. Axial T1-weighted MRI scan showing disappearance of the multiloculated contrast-enhanced lesions. therapy was administered including high-dose corticosteroids and antiepileptics. However, given their uncertain efficacy, antiplatelet (acetylsalicylic acid, clopidogrel) and vasodilatory (calcium channel blockers - propranolol, nimodipine; B-adrenoceptor blockers - verapamil) agents were added.

Based on a careful clinical status assessment, the initial high-dose corticotherapy administered to our patients (16-24 mg of dexamethasone per day) was gradually tapered. At the time of writing, patients 1 and 2 receive no corticotherapy, and patient 3 remains on a low maintenance dose of $2 \mathrm{mg}$ dexamethasone per day. All patients were initially administered antiplatelet therapy (acetylsalicylic acid: 100-200 mg per day), and patients 1 and 2 continue to receive it. Patient 1 was originally seizure-free on carbamazepine, but after a stroke-like attack, lacosamide was added. At the patient's request, lacosamide was withdrawn after stabilization of his clinical status. New antiepileptics (clonazepam, levetiracetam) were added to patient 3's chronic medication of valproic acid. As anxiolytic therapy, citalopram was administered in combination with bromazepam and alprazolam to Patients 1 and 2, respectively.

Patients 2 and 3 recovered completely. A SMART episode led to permanent worsening of neurological deficits in Patient 1, and a further complication was the radiationrelated basilar tip aneurysm, which was treated with endovascular coil embolization.

\section{CONCLUSION}

Stroke-like syndrome is a rare, delayed complication of brain oncotherapy. More than 100 cases have been published globally, but only cases with more favorable brain cancer diagnoses with a longer life expectancy have been reported ${ }^{3,6}$. For the first time, our case series presents three long-term survivors of GBM with stroke-like syndrome. A 5-year survival rate of 17\% for GBM patients under 50 years old was recorded in a randomized phase III study ${ }^{7}$, but only a 5-year overall relative survival rate of $6.8 \%$ in real clinical practice ${ }^{8}$. Originally, all our patients underwent tumor resection and chemoradiotherapy. All of them had acute MRI alterations, but two had imaging abnormalities before clinical manifestation. They presented with varied signs and symptoms (focal deficits, encephalopathy, seizures, and headache) (ref..$^{3,9}$ ). Strokelike syndromes developed within 2-5 days but resolved in 2-6 weeks. Diffusion-weighted MR and T2 brain perfusion abnormalities were detected in all patients, with focal MRI contrast enhancement due to blood-brain barrier disruption. Only Patient 1 had obscure thick gyriform cortical enhancement characteristic of SMART. Besides tumor recurrence, classic stroke, encephalitis, metabolic and mitochondrial disorders, and post-seizure swelling should be excluded ${ }^{9}$. The imaging indicated intensive MRI scanning and symptomatic medication (steroids, for their inflammatory and anti-edemic effects, supplemented by antiepileptics, vasoactive agents, etc.) for judicious 
management. It must be kept in mind that no imaging technique has $100 \%$ specificity and sensitivity, and both surgery and oncotherapy affect EEG and MRI results. Regarding the course, invasive procedures such as biopsy and tumor re-resection were still considered options.

The pathophysiology of stroke-like syndrome remains unclear, but a set of plausible causes has already been accepted. Compromised brain perfusion has been acknowledged in the literature as a process underlying syndrome progression ${ }^{10}$. Cranial irradiation alters neurogenesis, neuron morphology, and mitochondrial disorders, as well as causing endothelial damage, mainly to small vessels. In the following period, vascular dysfunction results in hypoxia of brain cells with impaired autoregulatory parameters and activates cerebral hyperexcitability. Conversely, seizure activity affects the previously injured brain microenvironment and potentiates inflammatory endothelial damage.

All stroke-like syndromes are diagnoses of exclusion. To avoid misinterpretation of imaging findings as GBM recurrence, and avert recall oncotherapy or redundant interventions, better understanding of delayed complications of brain tumor therapy is crucial. An antecedent event for the sudden onset of brain tissue destruction has not been elucidated. Close cooperation of neuro-oncology team members is essential, but neurological recovery is not always completed, and the recovery range of strokelike syndromes still remains unpredictable. To date, no knowledge of the possible parallel effects of surgery and systemic chemotherapy (temozolomide) on these syndromes' development has been acquired. Brain tissue reaction to cranial irradiation evolves in an individual pattern and without predictive markers. As no effective GBM treatment that spares normal brain tissue has been identified to date, cranial irradiation will continue to be the standard part of GBM treatment, and we will continue to encounter sequelae (such as stroke-like syndrome) for the foreseeable future. Hence, there are clear needs to characterize the sequelae, and identify ways to minimize both their frequency and severity.

\section{ABBREVIATIONS}

ALERT, Acute late-onset encephalopathy after radiotherapy; CNS, Central nervous system; CSF, Cerebrospinal fluid; FLT, 3'-deoxy-3'-[ ${ }^{18}$ F]fluorothymidine; GBM, Glioblastoma; IDH, Isocitrate dehydrogenase; MGMT, Methyl O6-methylguanine-DNA-methyltransferase; PET, Positron emission tomography; PCR, Polymerase chain reaction; PIPG, Peri-ictal pseudoprogression; SMART, Stroke-like migraine attacks after radiotherapy.

Authors contributions: OK: patient management, literature review, analysis of the radiologic data, initial draft manuscript preparation, concept and design of the study, analysis of the radiologic data, final approval of the version to be published; LH: conception and design of the study, final approval of the submitted version; $\mathrm{MH}, \mathrm{PK}, \mathrm{DF}, \mathrm{YK}$, MD: literature review, patient management; EC: analysis of the radiological data; $\mathrm{ZS}$ : literature review, evaluation and provision laboratory data, final approval of the submitted version of the manuscript; JD, MH, LT: literature review, initial draft manuscript preparation, evaluation and provision of laboratory and pathological data; OK, ZS: contributed equally to the work; All authors have read and approved manuscript.

Conflict of interest statement: The authors state that there are no conflicts of interest regarding the publication of this article.

\section{REFERENCES}

1. Sheikh S, Radivoyevitch T, Barnholtz-Sloan JS, Vogelbaum M. Longterm trends in glioblastoma survival: implications for historical control groups in clinical trials. Neuroocol Pract 2020;7(2):158-63.

2. Lim SY, Brooke J, Dineen R, O'Donoghue M. Stroke-like migraine attack after cranial radiation therapy: the SMART syndrome. Pract Neurol 2016;16(5):406-8.

3. Di Stefano AL, Berzero G, Ducray F, Eoli M, Pichiecchio A, Farina LM, Cuccarini V, Brunelli MC, Diamanti L, Condette Auliac S, Salmaggi A, Silvani A, Giometto B, Pace A, Vidiri A, Bourdain F, Bastianello $S$, Ceroni M, Marchioni E. Stroke-like events after brain radiotherapy: a large series with long-term follow-up. E. Eur J Neurol 2019;26(4):639-50.

4. Di Stefano AL, Berzero G, Vitali P, Galimberti CA, Ducray F, Ceroni $M$, Bastianello S, Colombo AA, Simoncelli A, Brunelli MC, Giometto B, Diamanti L, Gaviani P, Salmaggi A, Silvani A, Marchioni E. Acute late-onset encephalopathy after radiotherapy: an unusual lifethreatening complication. Neurology 2013;81:1014-7.

5. Urbanovska I, Houdova Megova M, Kalita O, Uvirova M, Simova J, Tuckova L, Buzrla P, Palecek T, Hajduch M, Dvorackova J, Drabek J. IDH1 mutation analysis by CADMA compared with SNaPshot assay and two immunohistochemical methods. Pathol Oncol Res 2019;25(3):971-78.

6. Biju RK, Dower A, Moon BG, Gan P. SMART (Stroke-Like Migraine Attacks After Radiation Therapy) syndrome: A case study with imaging supporting the theory of vascular dysfunction. Am J Case Rep 2020;28;21:e921795.

7. Stupp R, Hegi ME, Mason WP, et van den Bent MJ, Taphoorn MJ, Janzer RC, Ludwin SK, Allgeier A, Fisher B, Belanger K, Hau P, Brandes AA, Gijtenbeek J, Marosi C, Vecht CJ, Mokhtari K, Wesseling P, Villa S, Eisenhauer E, Gorlia T, Weller M, Lacombe D, Cairncross JG, Mirimanoff RO; European Organisation for Research and Treatment of Cancer Brain Tumour and Radiation Oncology Groups; National Cancer Institute of Canada Clinical Trials Group. Effects of radiotherapy with concomitant and adjuvant temozolomide versus radiotherapy alone on survival in glioblastoma in a randomised phase III study: 5-year analysis of the EORTC-NCIC trial. Lancet Oncol 2009;10:459-66.

8. Wen PY, Weller M, Lee EQ, Alexander BM, Barnholtz-Sloan JS, Barthel FP, Batchelor TT, Bindra RS, Chang SM, Chiocca EA, Cloughesy TF, DeGroot JF, Galanis E, Gilbert MR, Hegi ME, Horbinski C, Huang RY, Lassman AB, Le Rhun E, Lim M, Mehta MP, Mellinghoff IK, Minniti G, Nathanson D, Platten M, Preusser M, Roth P, Sanson M, Schiff D, Short SC, Taphoorn MJB, Tonn JC, Tsang J, Verhaak RGW, von Deimling A, Wick W, Zadeh G, Reardon DA, Aldape KD, van den Bent MJ. Glioblastoma in adults: A Society for Neuro-Oncology (SNO) and European Society of Neuro-Oncology (EANO) consensus review on current management and future directions. Neuro Oncol 2020;22(8):1073-113.

9. Black DF, Morris JM, Lindell EP, Krecke KN, Worrell GA, Bartleson JD, Lachance $\mathrm{DH}$. Stroke-like migraine attacks after radiation therapy (SMART) syndrome is not always completely reversible: a case series. Am J Neuroradiol 2013;34(12):2298-303.

10. Wilke C, Grosshans D, Duman J, Brown P, Li J. Radiation-induced cognitive toxicity: pathophysiology and interventions to reduce toxicity in adults. Neuro Oncol 2018;20(5):597-607. 